\title{
The perspectives of near-field interference microwave sensing based on the frustrated total reflection phenomenon
}

\author{
V.P. Belichenko* , A.S. Zapasnoy, A.S. Mironchev, A.V. Klokov, and E.V. Matvievskiy \\ National Research Tomsk State University, Russia, Tomsk, Lenin ave. 50, 634050
}

\begin{abstract}
A near-field microwave nondestructive diagnostics based on the total reflection phenomenon is described. The new type of schematic solution of device is proposed - a near-field interference microwave sensing system. The test diagnostics results in the conditions of metallized strips with breaks are presented.
\end{abstract}

\section{Introduction}

For a long time, unflagging interest has been shown in fundamental research of electromagnetic waves tunneling features. First of all, this interest was stimulated by the desire to find an explanation for the so-called "Hartmann paradox" during particle tunneling of a potential barrier [1]. The fact is that a direct solution based on experimental studies of the key problem of electrons tunneling transitions times through quantum barriers is extremely difficult. Therefore, a number of researchers, bearing in mind the formal similarity of the stationary Schrödinger equation and the Helmholtz equation, tried to find the answer based on the classical effects study of electromagnetic waves tunneling through macroscopic photonic barriers [1-3].

The experiments on tunneling of electromagnetic waves were carried out under conditions of frustrated total reflection (FTR). The FTR itself was realized [2] using two transparent for radiation $45^{\circ}-45^{\circ}-90^{\circ}$ prisms with a refractive index $n$ facing their large faces to each other (Fig. 1). The prisms, were cut from a cube of perspex with a side length of $400 \mathrm{~mm}$, having an index of refraction $n=1.605$ (the angle of total reflection $\left.\theta_{c}=38.5^{\circ}\right)$ at the frequency in question $(9.15 \mathrm{GHz})$. Microwaves with $\lambda_{\mathrm{o}}=32.8 \mathrm{~mm}$, generated in a klystron (2K25) were fed into a parabolic transmitter antenna guaranteeing quasiparallel beams. The beam spreading was less than $2^{\circ}$ as followed from $\sin \theta=\lambda_{\mathrm{o}} / 2 b n$ with diameter of antenna $b=350 \mathrm{~mm}$ and all beam components were in the range of total reflection. This was verified by measuring the transmission damping depending on the air gap between 0 and $50 \mathrm{~mm}$. The damping would be $1.8 \mathrm{~dB}$ in the case of normal reflection compared with authors measured $36 \mathrm{~dB}$ for the case of $\theta_{i}=45^{\circ}$ and a $50-\mathrm{mm}$ gap. The measured value of $7.2 \mathrm{~dB} / 10 \mathrm{~mm}$ were in agreement with the theoretical transmission. The signals have been picked up by a microwave horn and fed across an amplifier to an

* Corresponding author: bvp@mail.tsu.ru 
oscilloscope (HP 54825A). A metallic reflector placed at the base of the first prism to determine the position of the reflected beam in the case of geometrical optics.

In this case, the air gap $d$ was a uniform photon barrier for radiation beam 1 incident on the small face of prism I, and on the large face at an angle exceeding the angle of total reflection $\theta_{\mathrm{c}}=\arcsin (1 / n)$. Under these conditions, partial reflection of the radiation in the form of beam 2 occurs, partial tunneling with the Goos-Hänchen shift through the barrier in the form of an evanescent wave, and the subsequent propagation of radiation beam 3 in prism II (Fig. 1).

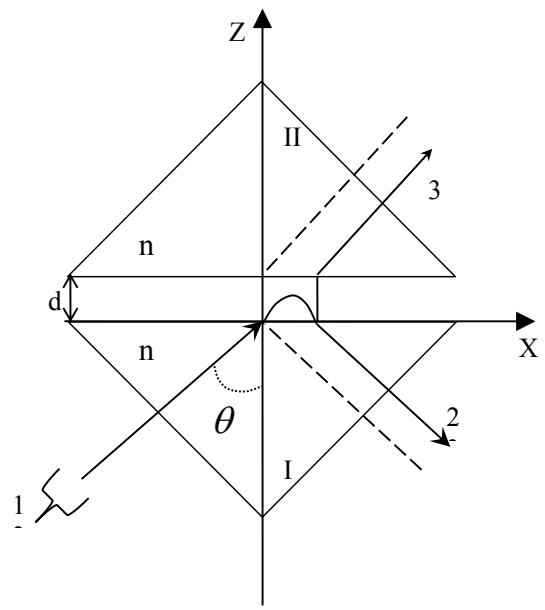

Fig. 1. Electromagnetic waves tunneling through uniform photon barrier [2].

It should be noted that the results of the studies met both support and quite severe criticism. The active discussions are continuing to this day. At the same time a certain value of these studies is connected, firstly, with a rather thorough development of the experimental base, and secondly, with the possibility of using the physical processes taking place to solve a completely different range of problems.

\section{Experimental arrangement}

In particular, this paper deals with the traditional technology of microwave spectroscopy modification using the FTR phenomenon. In a traditional technology framework, one rectangular prism is used, made of a material with a large refractive index. On the large face of the prism the object under study is placed with full contact with its surface. Under FTR conditions, the incident radiation will partially penetrate into the object in the form of an evanescent wave. In this case, the reflection coefficient of this radiation becomes dependent on the material parameters of the object under study. The extraction of material parameters from the FTR spectra is carried out after measurement and special processing of the reflected radiation spectra by the receiving system.

The modified technology is implemented using two identical prisms (Fig. 2). The object under study 4 is placed in the gap between the large faces of these prisms. The microwaves are radiated by a high-directional antenna 5 (Fig. 2). Along with antenna 5, another microwave antenna 6 of the same type is used (placing this antenna in different positions (Fig. 2) allows implement circuit solutions different in their characteristics). Both antennas are powered by the same microwave generator. This ensures the coherence of the fields emitted by the antennas. In addition, using the phase shifter included in the antenna 6 power supply circuit, the phase difference of the oscillations arriving at the inputs of the antennas 


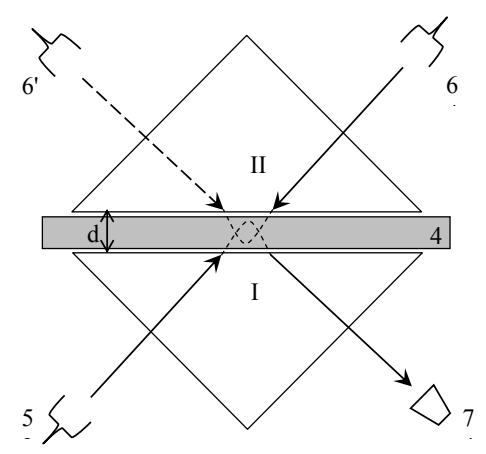

Fig. 2. Schematic of near-field interferential microwave sensing with using FTR.

5 and 6 is controlled. When the beams of electromagnetic waves are emitted by the antennas at angles exceeding critical values, an FTR takes place. In the gap between the large faces of prisms I and II, superposition of evanescent waves occurs, leading to the formation of a real interference energy flux [4]. It is essential to emphasize that the energy flux of an individual evanescent wave is purely imaginary. Flexible control of the influence of microwave radiation on the object 4 under study allows you to get more information from the measurement results of the receiving system 7 of the reflected radiation and their processing.

When developing a model that implements the proposed technology, we used information on the design and experiments methodology contained in [2]. In the transmitter part, the microwaves, generated by Agilent Technologies PNA-L Network Analyzer (N5230C) with the operating band of $10 \mathrm{MHz}-40 \mathrm{GHz}$ are fed into a coaxial conical horn sensor with aperture diameter $\mathrm{D}=70 \mathrm{~mm}$ to create a circular Gaussian like beam. This beam travels through small side of the prism I, as shown in Fig. 2, and immediately penetrates into a symmetrical system, consist of two same prisms. Both prisms are made by perspex with a dimension of $220 \times 220 \times 220 \mathrm{~mm}$ and have a refraction index with measuring value of approximate $n=1.5$ at the frequency of $4 \mathrm{GHz}$ in the system. In the receiver part, the reflection signals is detected by a similar coaxial conical horn sensor which is connected to Agilent Technologies PNA-L Network Analyzer.

\section{Experiments and results}

As an example the results of comparative tests of the technology are shown in Fig. 3a and 3b. This tests were aimed at identifying defects (gaps) in thin metal films. The measurements were carried out in the frequency range from 1 to $7 \mathrm{GHz}$, film breaks were $1,2,4$, and $6 \mathrm{~mm}$, and the films themselves were placed either on the face of a single prism or in the gap between the faces of two prisms. In order to significantly simplify the prototype design, a power divider was introduced into the antenna power circuit and measurements were made of the parameter $S_{11}$ (reflection coefficient) from a power divider, followed by the normalization of the measurement results to the measurement results for films without defects. As can be seen, even in this embodiment of the experiment, the proposed technology has advantages, at least due to the possibility of sounding in several frequency intervals.

Additional, more detailed measurements demonstrate, firstly, an improvement in sensitivity and resolution, and secondly, an increase in the reliability of sensing results due to the possibility of sounding in several frequency intervals (such a possibility, as it turned out during the experiments, was traditional technology practically does not provide). 


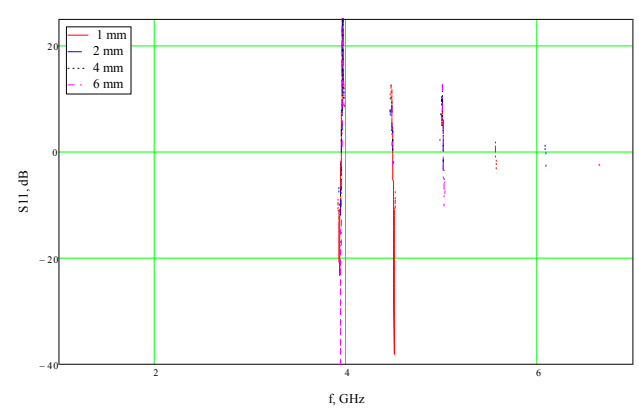

a) traditional technology

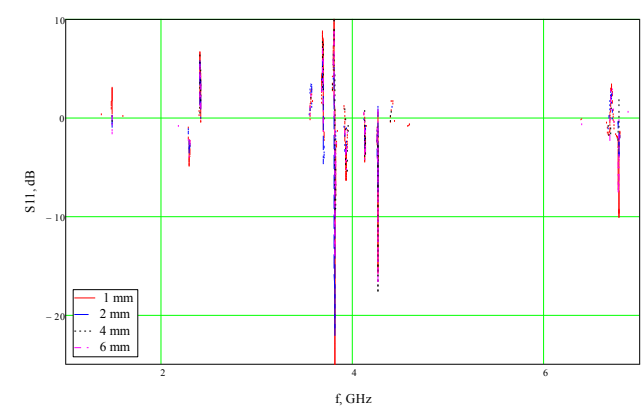

b) the modified technology

Fig. 3. The illustration $S_{11}$ parameter dependence on used technology of sensing.

\section{Conclusion}

In conclusion, we proposed the new type of diagnostics - the near-field interference microwave sensing technology. This technology is realizing in overlapping evanescent fields of two active sensors. The sensors are radiate monochromatic electromagnetic waves having a given initial phase shift. The said evanescent fields are generated in the small gap between the large faces of two dielectric prisms (where plane object under test is place) under frustrated total reflection conditions. Our test measurements are demonstrated an improvement in sensitivity and resolution, and an increase in the reliability of sensing results. We believe that the diagnostics of proposed type could be used in other spectral domain: terahertz, infrared, optical, ultrasound.

\section{Acknowledgments}

The work was performed within the framework of RSF, grant No. 18-75-10101.

\section{References}

1. A.B. Shvartsburg, Phys. Usp., 50, pp. 37-51 (2007)

2. A. Haibel, G. Nimtz, A.A. Stahlhofen, Physical Review E., 63, pp. 047601 (1-3) (2001)

3. D. Mugnai, A. Ranfagni, Lect. Notes Phys., 734, pp. 355-397 (2008)

4. S.A. Afanas'ev, D.I. Sementsov, Phys. Usp., 51, pp. 355-361 (2008) 